\title{
High Vegetable Diet
}

National Cancer Institute

\section{Source}

National Cancer Institute. High Vegetable Diet. NCI Thesaurus. Code C20048.

High consumption of vegetables and related micronutrients; exerts a moderate

protective effect on the risk of developing breast cancer. (from Medscape) 